\title{
Über die Gruppen linearer homogener Substitutionen vom Typus einer endlichen Gruppe.
}

Von

Alfred Loewy in Freiburg i. Br.

Im $53^{\text {ten }}$ Bande der Mathematischen Annalen*) habe ich den Begriff der Gruppe von endlich vielen linearen homogenen Substitutionen zu dem der Gruppe linearer homogener Substitutionen vom Typus einer endlichen Gruppe erweitert. Ein System \& linearer homogener Substitutionen in $n$ Variablen von nicht verschwindenden Determinanten soll eine Gruppe vom Typus einer endlichen Gruppe heißen, wenn das Produkt je zweier Substitutionen von $\mathbb{B S}$ wieder in $(\mathfrak{S}$ enthalten ist, $\mathfrak{B}$ neben jeder Substitution die reziproke enthält und die Gesamtheit charakteristischer Funktionen, die zu den Substitutionen von $\mathfrak{B S}$ gehören, nur für eine exdliche Anzahl untereinander verschiedener Werte verschwindet. Ist also

$$
A: \quad z_{i}^{\prime}=\sum_{k=1}^{k=n} a_{i k} z_{k} \quad(i=1,2, \cdots, n)
$$

irgend eine in (S) enthaltene Substitution, so soll nach der gegebenen Definition die Gesamtheit von charakteristischen Gleichungen:

$$
\left|-a_{j k}+\varrho \delta_{j k}\right|=\ddot{0} \quad\left(j, k=1,2, \cdots, n ; \delta_{j j}=1, \delta_{j k}=0(j \gtrless k)\right),
$$

die man erhält, wenn man für $A$ jede in $(S)$ enthaltene Substitution setzt, nur eine endliche Anzahl verschiedener Wurzeln $\rho$ besitzen. In dem früheren Aufsatz, dessen Kenntnis im folgenden nicht vorausgesetzt wird, war angenommen worden, daß die Gruppe (B) vom Typus einer endlichen Gruppe wenigstens eine Substitution besitzt, deren charakteristische Gleichung lauter voneinander verschiedene Wurzeln hat. Im vorliegenden Aufsatz soll die Theorie der Gruppen vom Typus einer endlichen Gruppe mit Hilfe eines neuen Satzes von W. Burnside völlig erledigt werden.

*) Zur Theorie der Gruppen linearer Substitutionen, Math. Ann. Bd. 53, S. 225-242. 


\section{$\S 1$.}

Das Burnsidesche Theorem, das wir im folgenden verwenden, lautet in unserer Terminologie: Jede irreduzible Gruppe linearer homogener Substitutionen vom Typus einer endlichen Gruppe ist eine Gruppe von endlich vielen Substitutionen.

Um die Lektüre des Burnsideschen Aufsatzes „On criteria for the finiteness of the order of a group of linear substitutions"*) nicht vorauszusetzen, erlaube ich mir, den Burnsideschen Beweis zu wiederholen. Nach einem Burnsideschen Fundamentalsatz**) läbt sich in jeder irreduziblen Gruppe (S) linearer homogener Substitutionen ein System von $n^{2}$ Substitutionen $A_{i}\left(i=1,2, \cdots, n^{2}\right)$ mit Koeffizienten $a_{\jmath k}^{(i)}$ derartig auswählen, dab die Determinante:

$$
\left|\begin{array}{cccc}
a_{11}^{(1)} & a_{12}^{(1)} & \cdots & a_{n n}^{(1)} \\
a_{11}^{(2)} & a_{12}^{(2)} & \cdots & a_{n n}^{(2)} \\
\vdots & & & \\
a_{11}^{\left(n^{2}\right)} & a_{12}^{\left(n^{2}\right)} & \cdots & a_{n n}^{\left(n^{2}\right)}
\end{array}\right| \neq 0
$$

wird. Ist $G$ mit Koeffizienten $g_{j k}(j, k=1,2, \cdots, n)$ irgend eine Substitution der irreduziblen Gruppe $\mathbb{B S}$, so gehören auch die Produkte $G A_{1}, G A_{2}, \cdots, G A_{n^{2}}$ der Gruppe $\mathbb{S}$ an. Ist \&s eine Gruppe vom Typus einer endlichen Gruppe, so kann die Summe der Diagonalglieder jeder Substitution von (S) nur eine endliche Anzahl verschiedener Werte annehmen; denn sie ist gleich der Summe der Wurzeln der charakteristischen Gleichung der betreffenden Substitution. Mithin werden die Summen der Diagonalglieder der Substitutionen $G A_{1}, G A_{2}, G A_{3}, \cdots, G A_{n^{2}}$ :

$$
\begin{aligned}
& \sum_{i=1}^{i=n} g_{1 i} a_{i 1}^{(1)}+\sum_{i=1}^{i=n} g_{2 i} a_{i 2}^{(1)}+\cdots+\sum_{i=1}^{i=n} g_{n i} a_{i n}^{(1)}=\mathrm{X}^{(1)} \\
& \sum_{i=1}^{i=n} g_{1 i} a_{i 1}^{(2)}+\sum_{i=1}^{i=n} g_{2 i} a_{i 2}^{(2)}+\cdots+\sum_{i=1}^{i=n} g_{n i} a_{i n}^{(2)}=\mathrm{X}^{(2)} \\
& \sum_{i=n} g_{1 i} a_{i 1}^{\left(x^{2}\right)}+\sum_{i=1}^{i=n} g_{2 i} a_{i 2}^{\left(n^{2}\right)}+\cdots+\sum_{i=1}^{i=n} g_{n i} a_{i n}^{\left(n^{2}\right)}=\mathrm{X}^{\left(n^{2}\right)}
\end{aligned}
$$

*) W. Burnside, Proceedings of the London Math. Society, ser. 2, vol. 3, p. $435-440(1905)$.

*) W. Burnside, On the condition of reducibility of any group of linear substitutions, Proceedings of the London Math. Society, ser. 2, vol. 3, p. 430-434 (1905). G. Frobenius und J. Schur, Über die Äquivalenz der Gruppen linearer Substitutionen, Sitzungsberichte der K. PreuBischen Akademie der Wissenschaften, Jahrg. 1906, S. 209. 
hierbei können die Größen $X^{(i)}\left(i=1,2, \cdots, n^{2}\right)$ nach dem Obigen nur eine endliche Anzahl verschiedener Werte annehmen. Mithin kommen, da die Determinante $\left|a_{j k \mid}^{(i)}\right| \neq 0$ ist, für die $n^{2}$ Koeffizienten $g_{i k}$, d. h. für die Koeffizienten sämtlicher Substitutionen der Gruppe $\mathcal{G}$, nur eine endliche Anzahl verschiedener Werte in Frage. Folglich enthält \&s nur endlich viele Substitutionen und ist eine endliche Gruppe.

Läbt sich eine beliebige Gruppe \&S linearer homogener Substitutionen auf irgend eine Weise durch eine Substitution $P$ von nicht verschwindender Determinante in eine ähnliche Gruppe $\mathfrak{A}=P(S) P^{-1}$ überführen, bei der die Matrix jeder Substitution die Form:

$$
\begin{aligned}
& \begin{array}{llllll}
\mathfrak{a}_{11} & 0 & 0 & 0 & \ldots & 0
\end{array} \\
& \begin{array}{llllll}
0 & \mathfrak{a}_{22} & 0 & 0 & \ldots & 0
\end{array} \\
& \left\{\mathfrak{a}_{11}, \mathfrak{a}_{22}, \cdots, \mathfrak{a}_{2 \lambda}\right\}: \quad \begin{array}{lllllll}
0 & 0 & \mathfrak{a}_{33} & 0 & \cdots & 0
\end{array} \\
& \begin{array}{llllll}
0 & 0 & 0 & 0 & \cdots & \mathfrak{a}_{\lambda \lambda}
\end{array}
\end{aligned}
$$

hat und $\mathfrak{a}_{11}, \mathfrak{a}_{22}, \cdots, \mathfrak{a}_{\lambda \lambda}$ Matrizes bedeuten, die irreduzible Gruppen erzeugen, so heiBt (S) eine vollständig reduzible Gruppe.

Ist $\mathfrak{A}=\left\{\mathfrak{a}_{11}, \mathfrak{a}_{22}, \cdots, \mathfrak{a}_{2, \lambda}\right\}$ eine Gruppe vom Typus einer endlichen Gruppe, d. h. haben die charakteristischen Gleichungen aller Substitutionen von $\mathfrak{A}$ nur eine endliche Anzahl untereinander verschiedener Wurzeln, so trifft für die irreduziblen Teilgruppen $\mathfrak{a}_{11}, \mathfrak{a}_{22}, \cdots, \mathfrak{a}_{2, \lambda}$ offenbar das gleiche zu. Nach dem angegebenen Burnsideschen Satze ist folglich jede von ihnen eine endliche Gruppe, mithin ist eine Gruppe $\mathfrak{A}=\left\{\mathfrak{a}_{11}, \mathfrak{a}_{22}, \cdots, \mathfrak{a}_{\lambda \lambda}\right\}$ vom Typus einer endlichen Gruppe eine Gruppe von endlich vielen Substitutionen. Ist \&S irgend eine Gruppe vom Typus einer endlichen Gruppe, so sind alle mit (S) ähnlichen Gruppen $P$ (S) $P^{-1}$ ebenfalls Gruppen rom Typus einer endlichen Gruppe; denn ähnliche Substitutionen haben die gleichen charakteristischen Funktionen. Hieraus folgt: Jede Gruppe rom Typus einer endlichen Gruppe, die vollständig reduzibel ist, ist eine endliche Gruppe. Nach einem zuerst ron Herrn Maschke*) bewiesenen Satze ist umgekehrt jede endliche Gruppe linearer homogener Substitutionen vollständig reduzibel.

*) H. Maschke, Beweis des Satzes, dab diejenigen endlichen linearen Substitutionsgruppen, in welchen einige durchgehends verschwindende Koeffizienten auftreten, intransitiv sind. Math. Ann. Bd. 52, S. 363. Vgl. auch G. Frobenius, Sitzungsberichte der Preußischen Akademie, Jahrg. 1899, S. 483. J. Schur, Neue Begründung der Theorie der Gruppencharaktere, Sitzungsb. d. Preuß. Akademie, 1905, S. 414. A. Loewy, Transactions of the American Math. Society, vol. 6, pag. 509. 
Wir haben daher das Theorem: Jede Gruppe linearer homogener Substitutionen vom Typus einer endlichen Gruppe ist stets und nur dann eine endliche Gruppe, wenn sie eine vollständig reduzible Gruppe ist.

\section{$\S 2$.}

Jede Gruppe linearer homogener Substitutionen läßt sich, wie ich in den Transactions of the American Mathematical Society, vol. 6, p. 504-533*) gezeigt babe, durch eine Substitution $A$ ron nicht verschwindender Determinante in eine ähnliche Gruppe $\mathscr{G}^{*}=A \mathbb{S S}^{-1}$ überführen, bei der die Matrix jeder Substitution die Form:

$$
\begin{array}{cccccc}
\mathfrak{S}_{11} & 0 & 0 & 0 & \ldots & 0 \\
\mathfrak{S}_{21}^{*} & \mathbb{S}_{22}^{*} & 0 & 0 & \ldots & 0 \\
\mathfrak{S}_{31}^{*} & \mathbb{S}_{32}^{*} & \mathbb{S}_{33}^{*} & 0 & \ldots & 0 \\
\vdots & & & & & \\
\mathfrak{G}_{\mu 1}^{*} & \mathbb{S}_{\mu 2}^{*} & \mathbb{S}_{\mu 3}^{*} & \mathbb{S}_{\mu 4}^{*} & \cdots & \mathbb{S}_{\mu,}^{*}
\end{array}
$$

hat und $\mathfrak{S}_{11}^{*}, \mathbb{S}_{22}^{*}, \cdots, \mathbb{S}_{\mu \mu}^{*}$ aufeinander folgende größte vollständig reduzible Gruppen bedeuten. Betrachtet man ähnliche Gruppen linearer homogener Substitutionen als nicht verschieden, so sind, wie ich bewiesen habe, die Gruppen

$$
\mathbb{B}_{11}^{*}, \mathbb{G}_{22}^{*}, \cdots, \mathbb{S}_{4^{*}}^{*}
$$

ihrer Reihenfolge nach als erste, zweite, usw. bis $\mu^{\text {to }}$ vollständig reduzible, zu \&S gehörige Gruppen eindeutig bestimmt und von der Wabl von $\mathfrak{G}^{*}$ unabhängig. Jeder Gruppe \&S kann man daher eine vollständig reduzible Gruppe $\left\{\mathbb{S}_{11}^{*}, \mathbb{S}_{22}^{*}, \cdots, \mathbb{S}_{\mu \mu}^{*}\right\}$ :

$$
\begin{array}{cccccc}
\mathbb{S}_{11}^{*} & 0 & 0 & 0 & \cdots & 0 \\
0 & \mathfrak{B}_{22}^{*} & 0 & 0 & \cdots & 0 \\
0 & 0 & \mathfrak{S}_{33}^{*} & 0 & \cdots & 0 \\
\vdots & & & & & \\
0 & 0 & 0 & 0 & \ldots & \mathfrak{S}_{\mu}^{*}
\end{array}
$$

zuordnen. Sieht man ähnliche Gruppen als nicht verschieden an, so ist die Gruppe $\left\{\mathfrak{G}_{11}^{*}, \mathbb{G}_{22}^{*}, \cdots, \mathbb{G}_{\mu, \mu}^{*}\right\}$ eindeutig bestimmt. Sie ist ferner zur Gruppe $\mathbb{S}$ homomorph. Jedem Element $R$ von $\mathbb{B}$ entspricht ein Element $V_{R}$ der Gruppe $\left\{\mathbb{S}_{11}^{*}, \mathbb{S}_{22}^{*}, \cdots, \mathbb{S}_{\mu \mu}^{*}\right\}$, und jedem Element der Gruppe $\left\{\mathbb{S}_{11}^{*}, \mathbb{S}_{22}^{*}, \cdots, \mathbb{S}_{\mu \mu}^{*}\right\}$, von der natürlich nur die voneinander

*) Über die vollständig reduziblen Gruppen, die zu einer Gruppe linearer homogener Substitutionen gehören. Vgl. auch den daran anknüpfenden Brief von Herrn L. Stickelberger, Transactions of the American Math. Society, vol. 7, pag. 509. 
verschiedenen Substitutionen beibehalten werden sollen, entsprechen ein oder mehrere Elemente von (S). Für je zwei Substitutionen $R$ und $S$ aus (S) wird das Produkt $V_{R} V_{S}=V_{R S}$, wenn $V_{R}, V_{S}, V_{R S}$ die zu $R, S$ und $R S$ zugeordneten Substitutionen der Gruppe $\left\{\mathbb{S}_{11}^{*}, S_{22}^{*}, \cdots, \mathbb{S}_{\mu_{\mu 1}}^{*}\right\}$ bedeuten.

Ist (S) eine Gruppe vom Typus einer endlichen Gruppe, so ist die

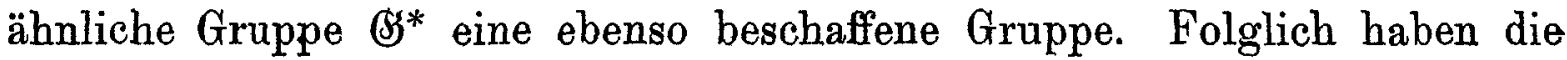
charakteristischen Funktionen aller Substitutionen von $\${ }^{*} *$ nur eine endliche Anzahl verschiedener Wurzeln; daher trifft dies auch für die vollständig reduziblen Gruppen $\mathfrak{S S}_{11}^{*}, \mathbb{S}_{22}^{*}, \cdots, \mathbb{S}_{\mu \mu}^{*} \mathrm{zu}$. Mithin sind diese nach dem am Schluß des $\S 1$ bewiesenen Satze endliche Gruppen. Folglich ist auch die Gruppe $\left\{\mathfrak{S}_{11}^{*}, \mathbb{S}_{22}^{*}, \cdots, \mathfrak{S}_{\mu \mu}^{*}\right\}$ eine Gruppe von endlich vielen Substitutionen. Ist umgekehrt die Gruppe $\left\{\mathbb{G}_{11}^{*}, \mathbb{S}_{22}^{*}, \cdots, \mathbb{S}_{\mu \mu}^{*}\right\}$ eine Gruppe von endlich vielen Substitutionen, so ist (S) offenbar eine Gruppe vom Typus einer endlichen Gruppe. Wir baben daher den Satz:

Die $\mu$ aufeinander folgenden größten vollständig reduziblen Gruppen

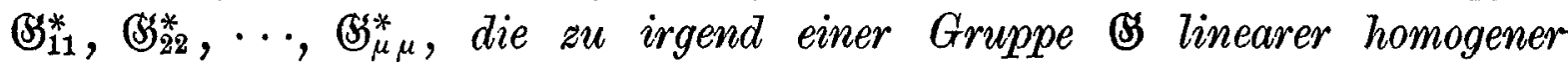
Substitutionen gehören, bestimmen eine der Gruppe homomorphe, vollständig reduzible Gruppe $\left\{\mathbb{S}_{11}^{*}, \mathbb{S S}_{22}^{*}, \cdots, \mathfrak{S S}_{\mu \mu}^{*}\right\}$; diese zu $\mathbb{S}$ gehörige Gruppe ist, wenn ähnliche Gruppen' als nicht verschieden angesehen werden, eindeutig bestimmt. Eine Gruppe (B) linearer homogener Substitutionen ist stets und nur dann eine Gruppe vom Typus einer endlichen Gruppe, wenn die homomorphe Gruppe $\left\{\mathbb{S}_{11}^{*}, \mathbb{S}_{22}^{*}, \cdots, \mathbb{S}_{\mu \mu}^{*}\right\}$ und folglich sämtliche Gruppen

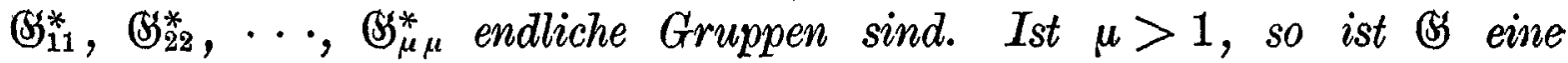
Gruppe von unendlich vielen Substitutionen, für $\mu=1$ ist (5) eine Gruppe von endlich vielen Substitutionen.

$\S 3$.

Die zu der Gruppe (S) homomorphe Gruppe $\left\{\mathbb{S}_{11}^{*}, \mathscr{S S}_{22}^{*}, \cdots, \mathbb{G}_{\mu \mu}^{*}\right\}$ sei mit 8 bezeichnet. (S) eine Gruppe vom Typus einer endlichen Gruppe, und \& bedeute den Komplex sämtlicher Substitutionen von \&S, die dem Einheitselement $E$ von 8 entsprechen. Alle Substitutionen $(5)$ von (S) sind dann Substitutionen der folgenden Form:

(a)

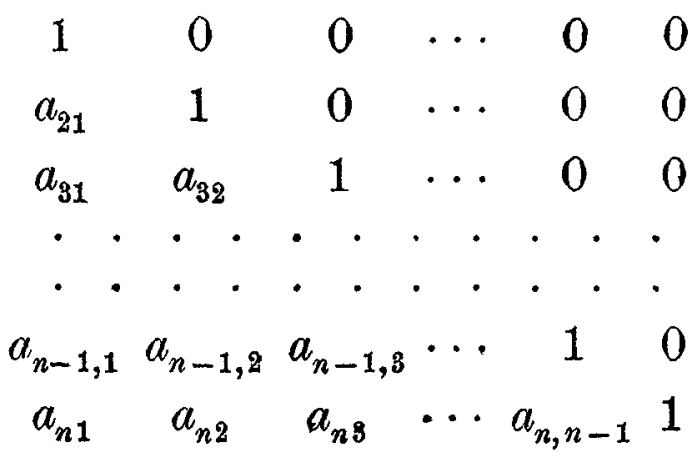


ähnlich; denn nur den Substitutionen der Form (a) von (S* entspricht die identische Substitution von 3. Die Substitutionen (a) von $\mathbb{S}^{*}$ bilden offenbar eine Untergruppe von (S*; mithin bestimmt auch der Komplex $\mathbb{E}$, dessen Elemente den Substitutionen (a) ähnlich sind, eine Untergruppe der zu (S) ähnlichen Gruppe (S).

Die zu (S) homomorphe endliche Gruppe $\left\{\mathbb{S}_{11}^{*}, \mathbb{S}_{22}^{*}, \cdots, \mathbb{S}_{\mu \mu}^{*}\right\}$ sei von der Ordnung $h$ und enthalte die $h$ voneinander verschiedenen Substitutionen $Z_{0}=E, Z_{1}, Z_{2}, \cdots, Z_{h-1}$. Dann lassen sich aus (S* $h-1$ Substitutionen $G_{i}^{*}(i=1,2, \cdots, h-1)$ auswählen, die den Substitutionen $Z_{i}(i=1,2, \cdots, h-1)$ von 8 entsprechen. $G_{i}(i=1,2, \cdots, h-1)$ seien die den Substitutionen $G_{i}^{*}$ aus $\mathbb{S}^{*}$ entsprechenden Substitutionen der Gruppe $\$$ S. Ich behaupte: Die Komplexe $\&$, \& $G_{1}, \& G_{2}, \cdots, \& G_{h-1}$ erschöpfen sämtliche Substitutionen von (G) $H$ sei eine beliebige Substitution von \&S und ihr entspreche die Substitution $Z_{k}$ von 8. Nach dem Obigen entspricht auch der Substitution $G_{k}$ ron (S) die Substitution $Z_{k}$ von 3. Folglich entspricht wegen des Homomorphismus der Substitution $G_{k}^{-1}$ von \&S die Substitution $Z_{k}^{-1}$ von 3 . Infolge des Homomorphismus von (S) und 8 ist ferner der Substitution $H G_{k}^{-1}$ der Gruppe (S) die Substitution $Z_{k} Z_{\bar{k}}^{-1}=E$ der Gruppe 3 zugeordnet. Mithin gehört das Element $H G_{k}^{-1}$ dem Komplex $\&$ an, und $H$ selbst ist eine Substitution aus dem Komplex $\& G_{k}$. Hiermit ist die aufgestellte Behauptung bewiesen. Der Komplex \& ist ferner eine invariante Untergruppe von \&. Einer Substitution von $H \& H^{-1}$ der Gruppe $(S)$ entspricht nämlich die Substitution $Z_{k} E Z_{k}^{-1}=E$ von 3 Mithin gehört jede Substitution $H \& H^{-1}$ von (S), wenn $H$ ein beliebiges Element aus (S) ist, dem Komplex (E) an. Wir haben daher den Satz:

Jede Gruppe of linearer homogener Substitutionen vom Typus einer endlichen Gruppe lesitzt eine invariante Untergruppe (5) von endlichem Index h. Die Zahl $h$ ist gleich der Ordnung der homomorphen, endlichen Gruppe $\left\{\mathbb{S}_{11}^{*}, \mathbb{S}_{22}^{*}, \cdots, \mathbb{S}_{\mu \mu}^{*}\right\}$, die zu $\mathbb{G}$ gehört. Der Gruppe \& entspricht

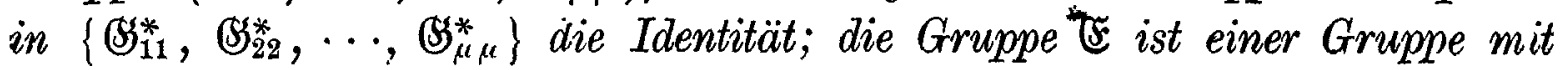
Shbstitutionen der Form:

$$
\begin{array}{cccccc}
1 & 0 & 0 & \cdots & 0 & 0 \\
a_{21} & 1 & 0 & \cdots & 0 & 0 \\
a_{31} & a_{32} & 1 & \cdots & 0 & 0 \\
\vdots & & & & & \\
a_{n 1} & a_{n 2} & a_{n 3} & \cdots & a_{n, n-1} & 1
\end{array}
$$

ähnlich. Die Gruppe $\mathbb{S}$ ist stets und nur dann endlich, wenn die invariante Untergruppe \& die identische Substitution ist. 
Die charakteristische Funktion jeder Substitution von $\&$ hat, wenn sie nicht die identische Substitution ist, wenigstens einen von 1 verschiedenen Elementarteilerexponenten. Hingegen haben die charakteristischen Funktionen sämtlicher Substitutionen einer endlichen Gruppe ausnahmslos Elementarteilerexponenten 1. Hieraus folgt:

Jede Gruppe vom Typus einer endlichen Gruppe ist stets und nur dann endlich, wenn die charakteristischen Funktionen aller Substitutionen der Gruppe ausnahmslos Elementarteilerexponenten 1 aufweisen.

Existiert eine ganze positive Zahl $r$, so daB alle Substitutionen einer Gruppe linearer homogener Substitutionen von $r^{\text {ter }}$ oder niedrigerer Ordnung sind, so hat die charakteristische Funktion jeder Substitution der Gruppe nur Wurzeln, die unter den Wurzeln der Gleichungen

$$
\varrho=1, \varrho^{2}=1, \cdots, \rho^{r}=1
$$

enthalten sind. Eine solche Gruppe ist mithin eine Gruppe vom Typus einer endlichen Gruppe. Beachtet man noch, dab die charakteristische Funktion jeder linearen homogenen Substitution endlicher Ordnung nur Elementarteilerexponenten 1 besitzen kann, so folgt nach dem zuletzt angegebenen Satz das zuerst von mir in den Math. Annalen, Bd. 53, S. 241 unter einer Einschränkung und dann allgemein von W. Burn si de(Proceedings of the London Math. Society, ser. 2, vol. 3, p. 438) bewiesene Theorem:

Kann man eine endliche positive ganze Zahl $r$ so finden, daß alle Substitutionen einer Gruppe von rer oder niedrigerer Ordnung sind, so ist die Gruppe endlich. Die Endlichkeit aller Gruppenelemente ist also die notwendige und hinreichende Bedingung für die Endlichkeit einer Gruppe linearer homogener Substitutionen.

\section{$\S 4$.}

Ob Gruppen vom Typus einer endlichen Gruppe endlich viele oder unendlich viele Substitutionen besitzen, läßt sich mit Hilfe der zugehörigen invarianten Hermiteschen Formen unterscheiden. Die Gruppe $\mathfrak{G}$ vom Typus einer endlichen Gruppe sei wie im $\S 2$ in die ähnliche Gruppe (\$):

$$
\begin{aligned}
& \begin{array}{lllll}
\mathfrak{S}_{11}^{*} & 0 & 0 & \ldots & 0
\end{array} \\
& \begin{array}{lllll}
\mathfrak{S H}_{21}^{*} & S_{22}^{*} & 0 & \cdots & 0
\end{array} \\
& \text { (S) } \\
& \mathbb{S}_{\mu 1}^{*} \quad \mathbb{S}_{\mu 2}^{*} \mathbb{S G}_{\mu 3}^{*} \ldots \mathfrak{S S}_{\mu \mu}^{*}
\end{aligned}
$$

transformiert. Die erste größte vollständig reduzible, endliche Gruppe (\$)* sei eine Gruppe von Substitutionen in $s$ Variablen. Wie jede endliche Gruppe linearer homogener Substitutionen führt auch $\mathbb{S}_{11}^{*}$ nach einem 
von Herrn E. H. Moore (Math. Annalen, Bd. 50, S. 213) und mir (Comptes Rendus 1896, S. 168) gleichzeitig gefundenen Satze eine definite Hermitesche Form von $s$ Variablen in sich über. Bei der Gruppe Ff* $^{*}$ und folglich auch bei der zu $\mathfrak{S f}^{*}$ ähnlichen Gruppe \&5 bleibt daher eine positive Hermitesche Form, d. h. eine solche, die abgesehen vom Werte 0 nur positive Werte annimmt, vom Range $s$ invariant.

Sei $s$ der Rang der positiven Hermiteschen Form höchsten Ranges, die bei allen Substitutionen einer Gruppe (S) vom Typus einer endlichen Gruppe invariant bleibt. Durch Einführung neuer Variablen kann man diese invariante positive Hermitesche Form in $y_{1} \bar{y}_{1}+y_{2} \bar{y}_{2}+\cdots+y_{s} \bar{y}_{s}$ transformieren; hierbei sind $\bar{y}_{1}, \bar{y}_{2}, \cdots, \bar{y}_{s}$ zu $y_{1}, y_{2}, \cdots, y_{s}$ konjugiert imaginäre Variable. Durch Einführung der neuen Veränderlichen geht die Gruppe $\mathbb{S}$ in eine ähnliche Gruppe $\mathfrak{S}$ über, deren Substitutionen die positive Hermitesche Form $y_{1} \bar{y}_{1}+y_{2} \bar{y}_{2}+\cdots+y_{s} \bar{y}_{s}$ invariant lassen. Die Substitutionen von $\mathfrak{H}$ seien von der Form:

$$
y_{i}=\sum_{j=1}^{j=n} h_{i j} y_{j}^{\prime} \quad(i=1,2, \cdots, n) .
$$

Da die Substitutionen ron $\mathfrak{H}$ die positive Hermitesche Form

$$
y_{1} \bar{y}_{1}+y_{2} \bar{y}_{2}+\cdots+y_{s} \bar{y}_{s}
$$

invariant lassen, müssen die Gleichungen bestehen:

$$
h_{1 s+t} \bar{h}_{1 s+t}+h_{2 s+t} \bar{h}_{2 s+t}+\cdots+h_{s s+t} \bar{h}_{s s+t}=0 \quad(t=1,2, \cdots, n-s) .
$$

Hierbei bedeuten die überstrichenen Größen die konjugiert imaginären zu den nicht überstrichenen. Folglich ist

$$
h_{i s+t}=0(i=1,2, \cdots, s ; t=1,2, \cdots, n-s) .
$$

Die Gruppe $\mathfrak{S}$ wird mithin von der Form:

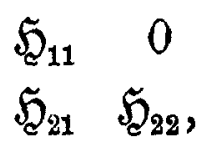

wobei $\mathfrak{S}_{11}$ ein System von Matrizes mit $s$ Zeilen und $s$ Kolonnen bedeutet und eine Gruppe definiert, die $y_{1} \bar{y}_{1}+y_{2} \bar{y}_{2}+\cdots+y_{s} \bar{y}_{\text {s }}$ invariant läßt. Jede Gruppe linearer homogener Substitutionen, die eine definite Hermitesche Form invariant läßt, ist vollständig reduzibel. ( $\nabla g l$. Transactions of the American Math. Society, vol. 6, 509.) Mithin ist $\mathfrak{F}_{11}$ eine vollständig reduzible Gruppe. Da $s$ die Rangzahl einer bei allen Substitutionen von \& invarianten positiven Hermiteschen Form höchsten Ranges sein soll, ist $\mathfrak{H}_{11}$, falls man ähnliche Gruppen als nicht verschieden ansieht, die eindeutig bestimmte, erste gröBte vollständig reduzible Gruppe, die zu (ff gehört. Wir haben daher den Satz: 
Jede Gruppe (S) linearer homogener Substitutionen vom Typus einer endlichen Gruppe läßt wenigstens eine positive Hermitesche Form vom Range $s \geqq 1$ invariant. Die Rangzahl jeder bei allen Substitutionen von (S) invarianten positiven Hermiteschen Form ist gleich oder kleiner als die Variablenzahl der ersten größten vollständig reduziblen Gruppe, die zu (S) gehört; diese Zahl wird auch mindestens einmal tatsächlich erreicht. IS ist stets und nur dann eine Gruppe von endlich vielen Substitutionen, wenn (\$S auch eine definite Hermitesche Form invariant läßt (d.h. eine solche, deren Rang gleich der Variablenzahl $n$ der Substitutionen von $\mathbb{S}$ ist oder die nur verschwindet, wenn ihre $n$ Paare konjugiert imaginärer Variablen sämtlich gleichzeitig Null gesetzt werden).

Der Zusatz „positiv" bei den im letzten Satze besprochenen invarianten Hermiteschen Formen ist wesentlich. Z. B. führt die Gruppe linearer homogener Substitutionen vom Typus einer endlichen Gruppe, die aus der Substitution $x_{1}^{\prime}=x_{1}, x_{2}^{\prime}=x_{1}+x_{2}$, ihren positiven und negativen Potenzen und der Identität besteht, die Hermitesche Form $x_{1} \bar{x}_{1}+i\left(x_{1} \bar{x}_{2}-x_{2} \bar{x}_{1}\right)$ in sich über. Diese hat den Rang 2, die Gruppe ist trotzdem nicht endlich; ihre erste größte vollständig reduzible Gruppe ist die aus der identischen Substitution $x_{1}^{\prime}=x_{1}$ bestehende Gruppe in einer Veränderlichen.

Freiburg i. Br., August 1906. 